\title{
FASCIOLOSIS: A MAJOR GLOBAL FOODBORNE ZOONOSIS
}

\author{
Hafiz Ishfaq Ahmad ${ }^{*}$, Khalid Mehmood ${ }^{2}$, Hui Zhang ${ }^{3}$, Muhammad Ijaz ${ }^{4}$, Rao Zahid Abbas ${ }^{5}$ and Riaz Hussain ${ }^{2}$ \\ 'Department of Animal Breeding and Genetics, University of Veterinary and Animal Sciences, Lahore, Pakistan \\ ${ }^{2}$ Department of Clinical Medicine \& Surgery, Faculty of Veterinary and Animal Sciences, The Islamia University of \\ Bahawalpur, 63100, Pakistan \\ ${ }^{3}$ College of Veterinary Medicine, South China Agricultural University, Guangzhou 510642, Guangdong, China \\ ${ }^{4}$ Department of Medicine, University of Veterinary and Animal Sciences, Lahore, Pakistan \\ ${ }^{5}$ Department of Parasitology, Faculty of Veterinary Science, University of Agriculture, Faisalabad-Pakistan \\ *Corresponding author: hafizishfaq93@outlook.com; khalid.mehmood@iub.edu.pk
}

\section{INTRODUCTION}

Agriculture sector plays a major role in the economy of Pakistan. It contributes approximately $11.4 \%$ to gross domestic product. This sector hires about $45 \%$ of labor force of the country and contributes to other segments of the growth economy (Khan et al. 2018). The livestock sector holds a unique place in the National Growth Agenda and is a net source of international revenue (Saleem et al. 2018). Throughout the history, smallholders have used livestock to satisfy their everyday needs for milk, food protection, and cash earnings.

Additionally, livestock is seen as a means of jobs at the rural level (Sargison 2020). It contributes significantly to poverty alleviation and continues to improve the socioeconomic status of our rural commonalities. In 201314 , livestock contributed roughly $11.9 \%$ to the national gross domestic product, adding $55.4 \%$ to agricultural value-added (Khan et al. 2020).

Parasitism is the major constraint to livestock production throughout the world (Das et al. 2018). The importance of worm diseases is magnified in underdeveloped countries, such as Pakistan, where $65.2 \%$ of the inhabitants live in rural areas and are economically dependent on livestock. Parasitism presents a significant challenge to the livestock economy of farmers (Abbas et al. 2014). Due to the broad spectrum of ultimate hosts, fasciolosis, also known as liver fluke disease, is very important among helminth pathogens (Chemale et al. 2010). Fasciolosis is a parasitic infection of the liver that affects both wild and domestic ruminants. It is caused by parasites of the genus Fasciola, which is found globally (Dawes and Hughes 1964). Economic losses occur due to cow deaths, feticide, stunted growth, decreased production of milk and meat, rejection of damaged liver and skinny cadaver, and animal treatment cost (Mehmood et al. 2017). Fasciolosis is responsible for a significant economic loss, triggered by $F$. hepatica in ruminants. The parasite has rapidly spread across Asia, Africa, and the Pacific. Though the disease is more prominent in young livestock, it may also affect older animals, impairing their fitness, growth rate, and production. Besides its common veterinary importance, fasciolosis caused by Fasciola hepatica and Fasciola gigantica has been a resurgent, affecting many human populations by spreading through zoonosis (Mas-Coma 2005).
The prevalence of Fasciolosis infestation is $49.01 \%$ in buffaloes. Between January and September, Fasciolosis was more widespread, and from October to December, it was least prevalent. Fecal tests showed a $65 \%$ prevalence of $F$. hepatica. In contrast, fecal egg counts showed that $F$. gigantica was more common in adult cattle than in calves (Khalil-ur-Rehman et al. 2009). The epidemiology and seasonal trend of flukes infection is determined by the availability of intermediate hosts and eating habits of final hosts. Life cycle of $F$. hepatica is complicated and consists of multiple stages that occur in the environment or the intermediate host (Mucheka et al. 2015). Metacercariae, the infectious stage, is encased and eaten by grazing herbivores. Temperature and rainfall are important factors affecting life cycle of $F$. hepatica and occurrence of infection (Karim et al. 2015). It has been identified in numerous studies that $F$. hepatica is endemic to cattle, buffaloes, dogs, goats and humans in Pakistan (Qureshi et al. 2005; Zafar et al. 2019).

Fasciolosis is a disease that can infect about $300 \mathrm{M}$ cattle 250M sheep globally (Mooney et al. 2009). The two liver flukes that are often reported to cause Fasciolosis in ruminants are F. hepatica and F. gigantica (Walker et al. 2008). Cattle that are infected usually have difficulties in gaining weight, and dairy cattle may have lower milk production and are more likely to develop metabolic diseases (Bekele and Getachew 2010). Fasciolosis costs the UK and Ireland more than $£ 18$ million annually. According to Mulcahy and Dalton (2001), the disease costs Sweden $€ 52$ million a year, or €299 per infected animal. Schweitzer determined that liver condemnations due to Fasciolosis cost Kenyan cattle slaughterers 0.26 million USD per year (Schweizer et al. 2007).

Fascioliasis causes substantial economic losses to pastoral agricultural communities and commercial animal farmers, estimated at US\$ 2 billion per year, through the death of infected cattle, liver condemnation, and productivity losses associated with reduced feed conversion quality (Sanchez-Vazquez and Lewis 2013). Fascioliasis is widespread in tropical areas, affecting up to $90 \%$ of cattle, and is considered the most significant helminths parasitic disease (Sanchez-Vazquez and Lewis 2013). Notably, humans may also contract Fasciola spp. and the infection is referred to as human Fasciolosis. It is estimated that $2.4 \mathrm{M}$ people are sick with this disease in 
more than 6o countries, and more than 180 million people are at risk worldwide (Rapsch et al. 2006). (Khalilur-Rehman et al. 2009) recorded a Fasciolosis infestation prevalence of $49.01 \%$ in buffaloes. Additionally, they found that the highest infestation occurred between January and September, while the lowest prevalence was between October and December. In contrast, Bhutto et al. (2012) found trematodes infestation in $4 \%$ of buffalo calves. Fasciola hepatica and, less often, Fasciola gigantica caused Fascioliasis. The large trematode's life cycle is illustrated in Figure 1.

Infected animals, such as humans, pigs, and buffalos, harbor adult flukes in their bile ducts, passing young Fasciola eggs in their feces. The next phase of the life cycle occurs in fresh water (Rapsch et al. 2006). After a few weeks, the eggs hatch into miracidium, which then infects a snail host. In the Snail, growth can be completed in 5 to 7 weeks under ideal conditions, and cercariae are then shed in the water. Cercariae lose their tails as they encyst to form metacercariae (infectious larvae) on water plants. Metacercarie, unlike cercariae, can live under damp conditions for extended periods because they have a hard-outer cyst wall (Zafar et al. 2019).

Evolution of Fasciola species in pre-domestication times

The uniquefeatures of Fasciolidae, with a total of just nine, largely geographically limited species scattered across three subfamilies, five monospecific genera, and only one multispecific genus indicate that this is an ancient tribe, with several members expected to have perished during its evolution (Mas-Coma et al. 2009). This evolution has resulted in the Fasciolids spreading across the Old World, with only one species, F. magna, having a Nearctic origin. In either case, the lack of Fasciolids in the Neotropical Region (all signs indicate that the presence of only $F$. hepatica in South America is a result of a recent human introduction) confirms the origin of Fasciolidae following the breakup of Gondwanaland into Africa and South America, approximately 90-100 million years ago (Dowd et al. 1994). The results of a recent phylogenetic analysis support the logical evolution of Fasciolidae members, including adult intestinal parasites with non-ramified caeca transmitted by planorbid snails, beginning in Africa and spreading to Asia and the rest of the Holarctic Region (Figure 2), with progressive adaptation to the liver microhabitat and transmission by lymnaeid snails (Lotfy et al. 2008). The origin of F. hepatica in Eurasia is widely known, owing to apparent preference of this Fsciolid for the lymnaeid Galba truncatula, which exhibits ecological characteristics associated with mild and cold climates (Mas-Coma et al. 2009). In terms of host origin, comparative evidence on infection, life span, egg shedding, and immunity suggest that sheep is more suited for $F$. hepatica than other host species. F. hepatica can live up to 11 years in sheep; adult flukes can produce a large number of eggs. However, there is no indication that sheep or goats develop immunity to $F$. hepatica. The disease is selflimiting in cattle. With the majority of flukes being

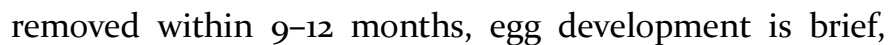
with high egg production lasting for just a few weeks, and resistance is gained during primary infection (Bekele and Getachew 2010; Mas-Coma 2005). These data indicate that $F$. hepatica originated in Eurasian ovicaprines, most likely in Ovis animals.

For F. gigantica, cattle tends to be more suited than sheep in terms of host origin, as it is more contagious and lasts longer in the former species. Resistance to $F$. gigantica has been found in sheep and $F$. gigantica has also been observed in goats (Anderson et al. 1999). Although the majority of $F$. gigantica adults survive less than a year in cattle, others may persist for at least 3-4 years (Schweizer et al. 2007), and faecal egg counts are up to $80 \%$ lower in buffaloes than in cattle with the exact infection dosage (Mehmood et al. 2017). These findings indicate that $F$. gigantica originated in African ruminant communities distinct from ovicaprines and somewhat resembling bovines (Ahmad et al. 2021).

\section{Life cycle}

Liver flukes infect a wide range of mammalian hosts and have a complicated life cycle (Valero et al. 2009). Humans are the accidental host of the genus Fasciola (LaPook et al. 2000). Metacercariae is the contagious type of this parasite, which burrows through the small intestinal wall and settles for a short time in the peritoneal cavity. Numerous liver fluke species are drawn to the biliary tree, whether intrahepatic or extrahepatic (Nyindo and Lukambagire 2015). The hepatic stage, which in $F$. hepatica is predicted to last for approximately 6-7 weeks (Jones et al. 2008), is characterized by mechanical penetration of the parenchyma from the liver capsule, with eventual entrance into the bile duct; this is the biliary stage. After sexual reproduction, parasites mature and lay eggs (Nyindo and Lukambagire 2015). In humans, metacercariae attain maturity within 3-4 months. Each adult worm produces an unusual number of eggs per day, depending on its definitive host. Sheep, pigs and black rats, according to reports, can extrude up to 25,00o, 12,00o and 2,150 eggs, respectively (Youssef and Uga 2014). An individual liver fluke has been reported to extrude approximately 40,00o eggs per day (Mucheka et al. 2015). These immature eggs are carried to the small intestine by bile medium, where they are mixed with feces. They are excreted in the pasture and undergo embryonic growth in definitive ruminant hosts under optimum humidity and temperature. Since aquatic bodies play a critical role in the development of liver fluke larvae, hatching is determined by environmental factors such as light, temperature, and humidity (Tavil et al. 2014). Via constructive chemotactic and phototactic motions, the emerging free-swimming ciliated miracidia are designed to locate an essential Limnaeid snail intermediate host in less than 24 hours (Mucheka et al. 2015). They mature into sporocysts by mechanically penetrating the body wall and tissues of their snail hosts with their sharp stylets and proteolytic enzymes (Tavil et al. 2014). Mother rediae are produced by the further metamorphosis of the sporocysts, 
which grow into daughter rediae. In the snail host, these rediae metamorphosize into cercariae, which can passively infect suitable vertebrate hosts and humans, who drink infested water (Nyindo and Lukambagire 2015). Relative humidity of more than $65 \%$, rainfall more than $100 \mathrm{~mm}$ annually, and an atmospheric temperature of 25$30^{\circ} \mathrm{C}$ have been identified as favorable conditions for cercariae growth and shedding (Gu et al. 2012).

\section{Epidemiology of fascioliasis}

Fasciolosis is widespread (Figure 1) in a variety of Asian countries, including the Middle East (Saudi Arabia, Russia, Pakistan, Thailand, Iraq, Iran, China, Iraq, Turkey, Vietnam, Japan, Korea, Iran, Philippines, Cambodia, Bangladesh, and Nepal) (Mehmood et al. 2017). Prevalence of Fasciolosis has been identified in thirteen countries and forty-one trials, ranging from $0.71-69.2 \%$ in cattle to $0.0-47.0 \%$ in goats (Table 1 and Figure 1 ). Table 1 shows the prevalence of Fasciolosis in Asian countries from 2000 to 2015. Iran and Pakistan have the most significant number of records among Asian countries. The bulk of experiments containing a greater number of animals reported a lower prevalence in comparison to those involving a short period and a smaller number of animals. Although the prevalence of Fasciolosis in Bangladesh has been reported to range between 14.28 and $21.54 \%$ in goats (Mehmood et al. 2017; Sanchez-Vazquez and Lewis 2013), and no data in other output species are known, the higher prevalence of parasite in this country can be due to the moist conditions and high rainfall, which are ideal for survival and reproduction of snails (Ahmed et al. 2007). According to a regional information method for assessing Fasciolosis, the prevalence of parasites in cattle from Cambodia has been estimated to be up to $20 \%$ (Dorny et al. 2011). Fasciolosis may affect $28 \%$ of cattle in Cambodia, areas with high and average risk concentrated in southern and central Cambodia (Dorny et al. 2011). According to a study conducted on sheep in China, the overall prevalence of liver fluke was estimated to be $28.5 \%$, indicating that management strategies should be applied (Wang et al. 2006). In India, various figures have been recorded for sheep (2.78$8.98 \%)$, goats $(2.35-15 \%)$, cattle (10.79\%), and buffaloes (13.9\%) (Mehmood et al. 2017). The high prevalence of these parasites may be attributed to suitable weather conditions for snails, the abundance of low-lying, wellirrigated marshy land and pastures near water sources, which are ideal for breeding of intermediate hosts. In Iran, prevalence of Fasciolosis has been reported in goats (o.20-4.4\%), cattle (0.71-81.5\%) and sheep (0.35-31.25\%). There is a major seasonal difference in the prevalence of Fasciola spp (Bhutto et al. 2012). Fluke egg hatching and snail replication involve high rainfall and cool temperatures, occurring in spring and autumn. Fasciolosis has been reported in Iraq with a rate of $0.36 \%$ in sheep, $0.14 \%$ in goats, $1.27 \%$ in cattle, and $2.08 \%$ in buffaloes (Kadir et al. 2012), although it has been reported at a rate of up to $2.00 \%$ in Japan. It infects $1.10 \%$ in the Korean goat population, although it was the largest in buffalo and goat populations in Nepal compared to all other Asian countries (Mehmood et al. 2017). Numerous tests have been performed in Pakistan, and the average prevalence of Fasciolosis in sheep is $6.50-29.99 \%$, in goats it is $0.66-28.75 \%$, in cattle it is $19.30-20.42 \%$, and in buffaloes it is $14.71-30.50 \%$ (Mas-Coma et al. 2009). The causes for the rise in Fasciolosis infection rates may be the farmers' socioeconomic status or the creation of tolerance due to the inappropriate use of anti-parasitic drugs at insufficient doses for a prolonged period. In Saudi Arabia, Fasciolosis affects $13.50 \%$ of sheep and $52.90 \%$ of cattle (Sanad and Al-Megrin 2005). The higher incidence in cattle may be attributed to local farmers' lack of awareness about control measures. Numerous experiments conducted in Turkey indicate that Fasciolosis is present in up to $16.20 \%$ of sheep, $0.80 \%$ of goats, and $69.20 \%$ of cattle. Owing to less swampy regions and global warming, Turkey has a lower incidence of Fasciolosis than the nearby countries (Mehmood et al. 2017).

\section{The transmission pattern of Fascioliasis}

It is worth noting that there is remarkable variability of human Fascioliasis in epidemiological scenarios and dissemination rates worldwide (Ahmad et al. 2021). It may be inferred that well-known circumstances and trends do not always sufficiently account for disease attributes in a given region (Mehmood et al. 2017). Thus, when tackling an unstudied endemic area, the scenarios and dynamics of human infection outlined above must always be regarded as starting points. Only after an accurate evaluation of the epidemiology and transmission characteristics of the new region has been undertaken, suitable protection mechanisms for the zone in question can be planned (Nyindo and Lukambagire 2015). It is critical to emphasize on the relationship between the lymnaeid vector species and the propagation pattern in question. Lymnaeids exhibit marked ecological and behavioral variation among species. Factors such as water selection temperature thresholds, habitat type, population dynamics, seasonality, and liver fluke infection susceptibility are critical for Fascioliasis (MasComa 2005). All of these facts suggest that lymnaeids can serve as excellent disease markers, help differentiate between the various human Fascioliasis scenarios and trends, and thus as determinants for developing effective control strategies (Kadir et al. 2012).

A very high-altitude trend is linked with only $F$. hepatica transmission in Andean countries through imported Galba truncatula; within this group, two sub-patterns can be differentiated based on seasonal and psychological characteristics (Mas-Coma 2005). The altiplanic pattern is characterized by year-round transmission (e.g. in the Northern Bolivian Altiplano and the Puno Altiplano). The valley pattern is seasonal and varies in severity due to altitude as in the Peruvian valleys of Mantaro and Cajamarca (Mehmood et al. 2017). A Caribbean insular trend, with smaller yet more regular outbreaks in human hypo-endemic regions and lymnaeid species except 
Table 1: Global prevalence range (\%) of Fasciolosis in ruminants reported in different continents from 2000 to 2020 (Mehmood et al. 2017; Opio et al. 2021).

Sr. No. Countries

Continent

Studies Prevalence in different species

$1 \quad$ Egypt, Kenya, Chad, Ethiopia, Nigeria, Sudan, Tunisia, Africa Sheep Cattle Buffaloes Uganda, Zimbabwe, Tanzania, Zambia

2 Colombia, Argentina, Peru, Brazil, Mexico

America

$\begin{array}{lllll}31 & 0.28-68.4 & 0.19-73.7 & 1.2-91.0 & 9.73-33.7\end{array}$

India, Bangladesh, China, Iraq, Cambodia, Pakistan, Asia

Nepal, Iran, Japan, Korea, Vietnam, Saudi Arabia, Turkey

$4 \quad$ Australia, Papua New Guinea

Australia/Oceania 03 region

5 England, Italy, Belgium, Denmark, Spain, Germany, Europe Ireland, Switzerland, Poland, Sweden, Wales

$\begin{array}{lllll}10 & 24.5-100 & 8.87-100 & 3.0-66.7 & 11.4-24.4 \\ 41 & 0.0-47.0 & 0.35-31.4 & 0.71-69.2 & 2.08-68.0\end{array}$
$\begin{array}{llll}0.0-47.0 & 0.35-31.4 & 0.71-69.2 & 2.08-68.0\end{array}$

$(18.2)$

$5 \cdot 5-52.2 \quad 26.5-81.0$

23

0.0-0.8 $3-83.3 \quad 0.12-86.0 \quad-$

(): just single study no range is available.

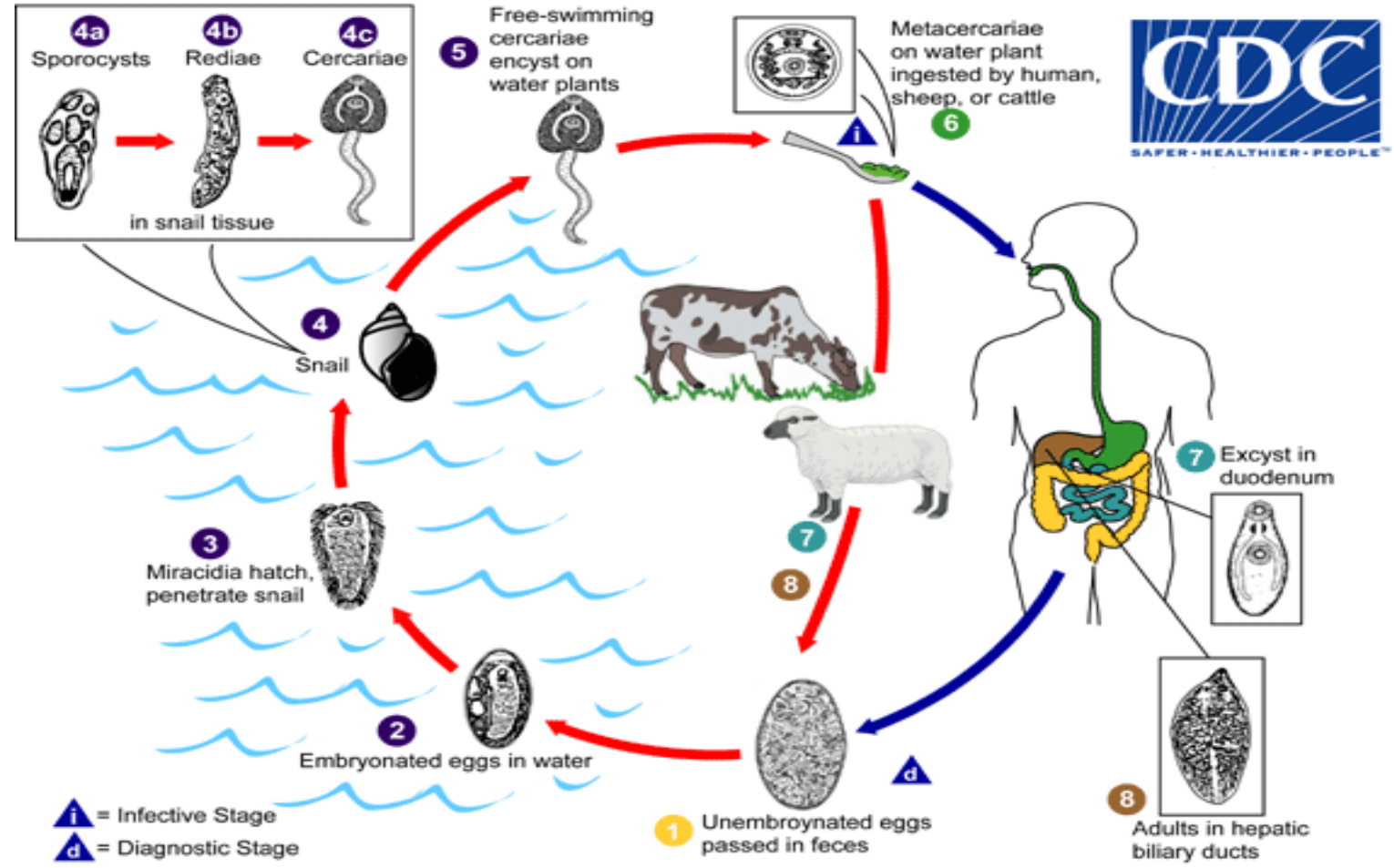

Figure 1: The life cycle of large liver fluke (F. hepatica) (Hurtrez-Boussès et al. 2001).

primary vector species, is involved in transmission, as in the Pinar del Rio Province in Cuba. A trend characteristic of Afro-Mediterranean lowlands involves alternating $F$. hepatica and F. gigantica, many Galba fossaria and Radix lymnaeids, as well as secondary transmitting Pseudosucinea (Ahmad et al. 2021). A trend was seen in surrounding areas of Caspian Sea, including human hypoendemic zones, in which significant epidemics occasionally reach 10,000 people, including $F$. hepatica and $F$. gigantica, as well as many Galba fossaria, Radix, and stagnicoline lymnaeids (Iran) (Lotfy et al. 2008).

\section{Diagnosis and treatment}

In endemic areas, if the clinical picture is highly indicative, it is often unremarkable. As a result, coprological procedures, such as looking for larvae, or examining adult worms vomited or passed out in feces are used for diagnosis (Le et al. 2008). In the GenBank database, two sequences corresponding to Fasciola buski's
18S rRNA gene are available. Recently, Vietnamese child raise sequence of a fluke was used to verify the diagnosis. When the alleles were compared, only two nucleotide substitutions were detected (Nguyen et al. 2009). A number of drugs have been evaluated for the treatment of the disease in different ways. Tetrachloroethylene was discovered as an effective antibiotic (Karim et al. 2015) and became the preferred drug. Thiabendazole, levamisole and mebendazole are ineffective (Kelly et al. 1977). In the later studies, praziquantel was found to be effective, even in severe cases, resulting in prescribing a single $15 \mathrm{mg} / \mathrm{kg}$ dosage as the preferred therapy (Coles 1986), even though this drug was unable to save the life (Maqbool et al. 2002). In experiments on pigs, relative efficacy of triclabendazole, rafoxanide and oxyclozanide for the treatment of Fascioliasis was investigated. It is worth mentioning that triclabendazole has a high degree of efficacy (97.12\%), followed by oxyclozanide (93.27\%) and rafoxanide $(83.17 \%)$, all of which are completely safe (Chhabra and Singla 2009). 


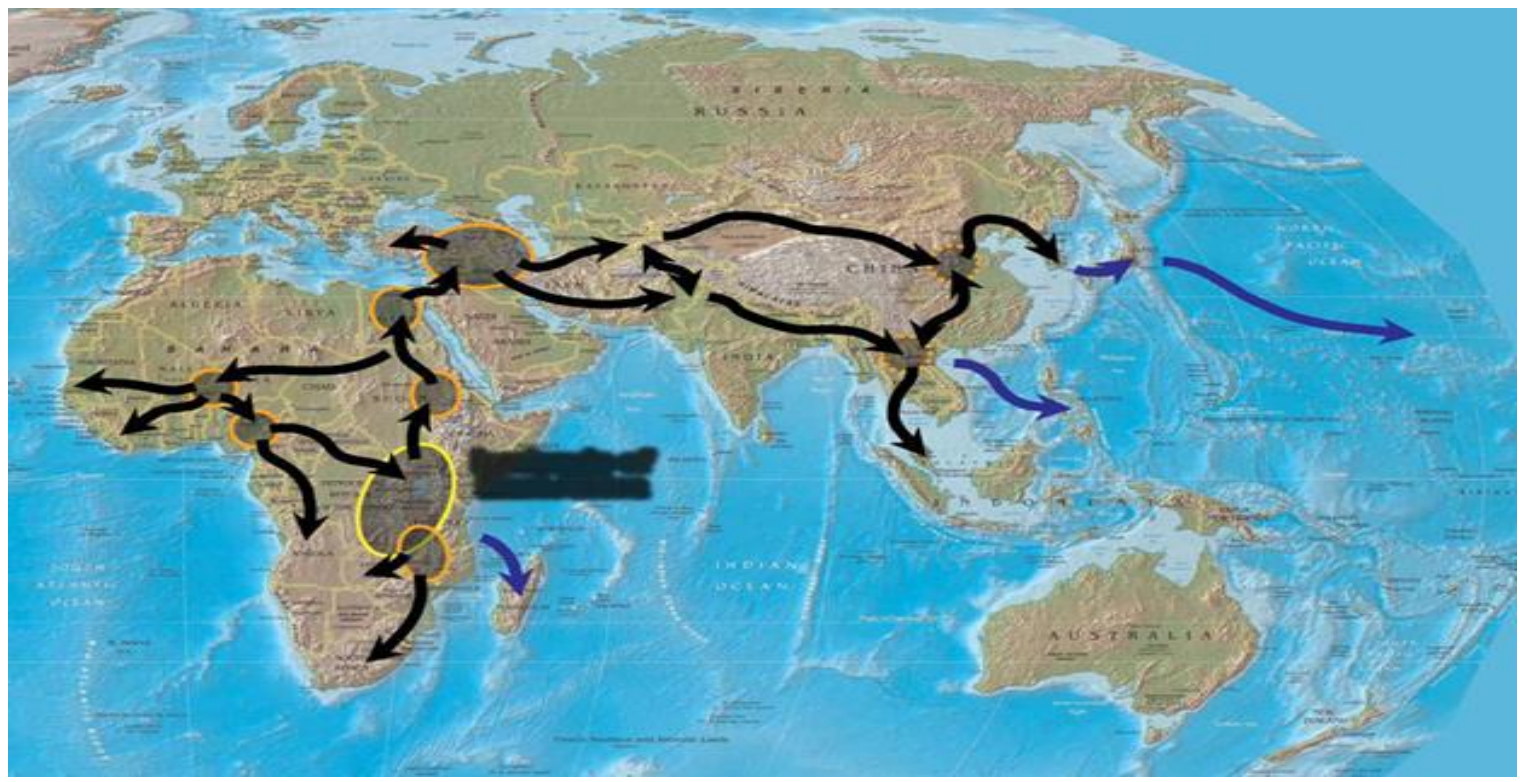

Figure 2: Geographical spread routes followed by F. gigantica in the post-domestication period (Mas-Coma et al. 2009).

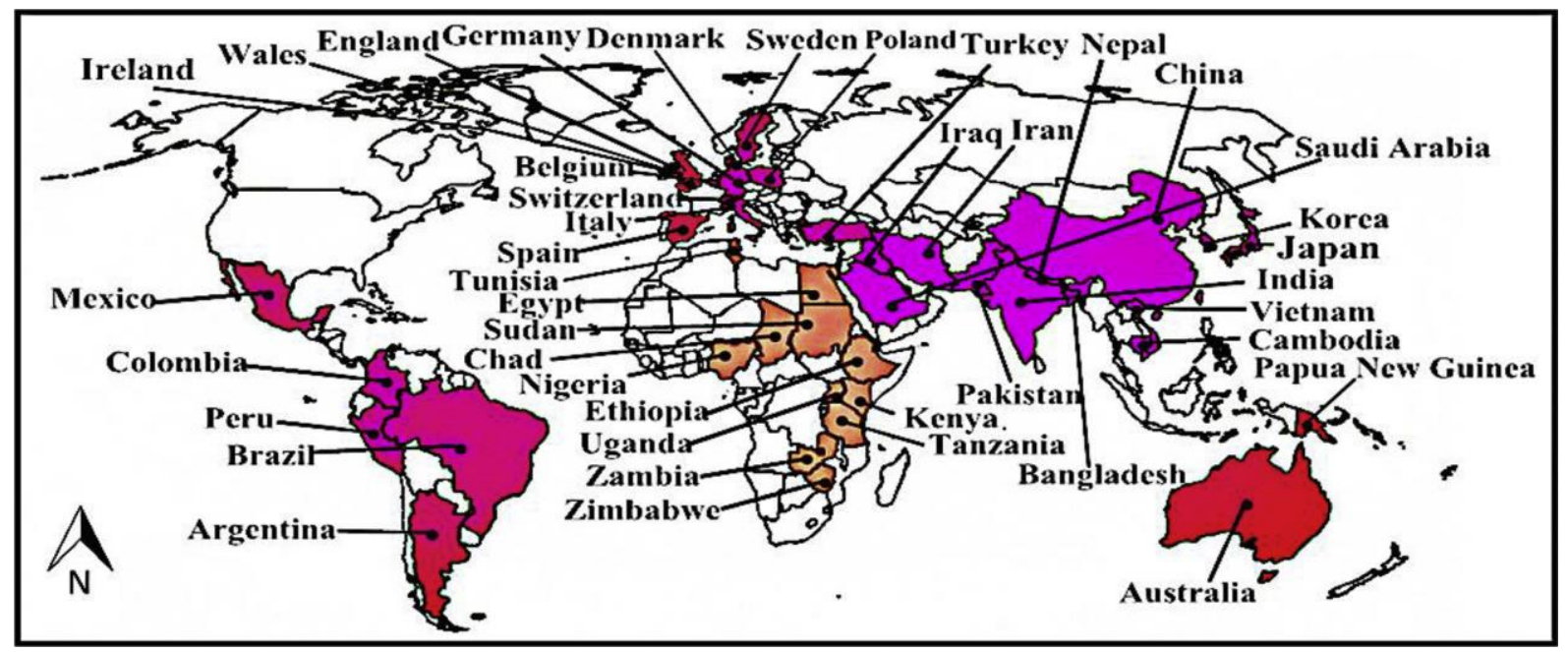

Figure 3: Global prevalence of Fasciolosis in ruminants reported (2000-2015) in different countries (Mehmood et al. 2017).

\section{A neglected disease}

Fascioliasis caused by certain species of macroscopic and leaf-like digenetic liver flukes of the genus Fasciola is a medically necessary, primitive foodborne neglected zoonotic disease (Hugh-Jones et al. 1995). In 1379, a French scientist, Jehan De Brie identified the first known parasite, F. hepatica (ME et al. 1995). A pathologist and resident physician in Calcutta, Professor James McConnell, in 1874, discovered a Chinese human liver fluke called Clonorchis Sinensis after an autopsy on the body of a twenty-year-old carpenter (Nyindo and Lukambagire 2015). At the moment, alleged hybrids between $F$. hepatica and $F$. gigantica are being studied to determine their actual taxonomic status (Jones et al. 2008).

This is because an estimated $2.4 \mathrm{M}$ people in more than 70 countries worldwide suffer from Fascioliasis. These trematodes infest every continent, and 18oM people are at risk ( $\mathrm{ME}$ et al. 1995). Moreover, it is estimated that $F$. hepatica infects over 250M sheep and 30oM cattle worldwide, along with $F$. gigantica, causing an estimated US $\$ 3 \mathrm{~B}$ in economic damage per year (Hugh-Jones et al. 1995). Recently, the global incidence of human Fascioliasis has increased significantly (Mehmood et al. 2017), with a close link to a high infection rate in definitive ruminant hosts. The transmission of parasite is carried out by a diverse population of universal aquatic snails in Limnaeidae. For example, Hinkleyia caperata, Austropeplea tomentosa, Galba truncatula, Radix rubiginosa, Stagnicola corvus, and Pseudosuccinea columella have been recorded as endemic to Australia, North America, Europe, Africa, Asia, and South America, respectively (Vázquez et al. 2018). Fascioliasis caused by $F$. gigantica is endemic in tropical and subtropical lowlands. As a result, more disease cases are being recorded in a more significant portion of Sub-Saharan Africa (SSA), where suitable snail intermediate hosts occur naturally (Kelly et al. 2019).

Due to the high prevalence of Fasciolid infection in humans, this disease has been underestimated. Between 
the 1950 os and 1990s, the disease gained attention in France due to the hundreds of thousands of patients hospitalized during that period (Corvo et al. 2013). Fascioliasis was one of the most untreated illnesses on a global scale. Although the situation has dramatically changed since the 1990s, the often-used term sheet and cow disease' continues. This presents challenges in convincing political and health authorities of vital existence of human infection in many countries and the importance of introducing prevention measures, expert research and health literature (Jones et al. 2008). Thus, Fascioliasis receives no coverage in textbooks covering various diseases, such as tropical or human parasitic diseases. Researchers make fewer attempts to update knowledge on secondary infections than on primary ones. At times, research papers published in high-impact technical journals include inaccuracies, misunderstandings, or mistakes (Le et al. 2008).

\section{Immediate concern about Fascioliasis}

Global understanding of zoonoses and a one-health solution are by far the most systematic approaches for the control of Fascioliasis (Phiri et al. 2005). Sustaining actions, including trade and travel restrictions, are expected to prevent infected animals transmission between countries. Additionally, enhanced food and water hygiene systems are critical parts of the monitoring scheme (Hammami et al. 2007). Stakeholder engagement and political support for these policies are essential for their successful implementation. Complete triclabendazole resistance cases (Islam and Ripa 2015) should be thoroughly reviewed to find out alternate treatment or solutions.

Additionally, vaccine development should be a focus of future studies. Multiple trematodes or intestinal parasite infections complicate the early diagnosis of Fascioliasis (Ahmed et al. 2007). Delayed and missing diagnosis, principally among the young ones, magnifies adverse effects of the disease (Karim et al. 2015). While Fascioliasis has been recognized as a disease of human importance, it is mainly considered as an animal disease, most notably of cattle and sheep. Lack of knowledge is a significant impediment to effectively managing human Fascioliasis (Bekele and Getachew 2010). Unfortunately, Fascioliasis in humans is not a recognized and reportable disorder in a large proportion of the world's least developed countries, which are plagued by hunger and infectious diseases. Sensitization and awareness are critical first steps in any proposed intervention strategy. Fortunately, there is no death identified due to human Fascioliasis infection to date. As a result, the disease is predictably assigned a low emergency health priority, making it one of the most underestimated tropical diseases (Mehmood et al. 2017).

Additionally, a slightly higher prevalence in school-aged girls has been reported. Thus, the morbidity and Disability Modified Life Years (DALY) effects of the disease are of great significance (Moje et al. 2015). The morbidity associated with chronic disease remarkably leads to reduced life expectancy, poor quality of life and economic performance. The adult worms can survive for more than ten years in a suitable host (Mas-Coma et al. 2009).

\section{Fascioliasis as an emerging zoonosis}

The World Health Organization has stressed the critical nature of foodborne fluke infections and the critical nature of controlling measures (ME et al. 1995). Plantborne trematodes were newly added to the Institute of Food Technologists' Specialist Council on Food Safety and Nutrition (Hugh-Jones et al. 1995). In November 2004, the Third Global Meeting of the Partners for Parasite Control at the WHO Headquarters in Geneva, Fascioliasis and other foodborne trematode diseases were added to the significant list of helminthiases that have a direct effect on human health (Nyindo and Lukambagire 2015). Additionally, it appears that current climate and global patterns affect some snail-borne helminthiases that depend on the environment for their transmission. Fascioliasis is an excellent example of a parasitic disease emerging/re-emerging in various countries due to a mixture of natural and human-made changes (Nguyen et al. 2009).

\section{Prevention and control}

Regulations can be made for handling livestock, preventing augmentation, and adopting new pig farming techniques (Schweizer et al. 2007). Individual avoidance is straightforward: prevent use of foods obtained from freshwater plants. However, application of such regulation is incredibly difficult when century-old rituals are involved (Graczyk and Fried 2007). Infections are most likely to spread within families, since food storage, and eating practices are passed on over generations (Hurtrez-Boussès et al. 2001). Additionally, due to their low cost and availability, aquatic plants are a common food source (Hugh-Jones et al. 1995). Techniques for preventing contamination include preventing the use of human feces as waste, abandon defecation, and washing pig excreta into nearby water bodies (Le et al. 2008). Desiccation and direct solar radiation destroy metacercariae. The prevention approach should be focused on sustained outreach, emphasizing the importance of extensively cooking freshwater plants, immersing fruit and plants in boiling water for a few minutes, and use of boiled water in areas without access to purified water (Jones et al. 2008).

\section{Future challenges}

There have been significant advancements in the diagnosis, surveillance, and treatment of Fascioliasis. Nonetheless, under-developed countries, primarily lowincome populations, cannot benefit from these advancements due to poverty. With pathogens, civil strife, and competition for scarce capital, it is unsurprising that these areas have too few cases of human 
Fascioliasis. Control initiatives should continue with extensive education and sensitization campaigns about the effects and magnitude of Fascioliasis in animals and humans. The new "One Health Integrated Global Approach to Disease" is the most systematic and participatory response available, not only for human Fascioliasis but also for most zoonotic diseases in general. A classic indicator of the challenges it discusses is the latest movement to "Go Eco" as a sustainable alternative to the current artificial lifestyle. This has resulted in extraordinary growth in the intake of fresh, raw/green vegetables and fruits. This is contradicted by a lack of funding for water protection, fertilizer-pesticide controls, and waste management. The use of unmonitored and unprocessed fresh vegetables has aided in the spread of plants/foodborne liver flukes such as Fascioliasis and a variety of other health problems. Clinical trials to assess confirmed cases of Fasciola bithionol and triclabendazole resistance are urgently needed. Further production of chemotherapeutic agents, such as Mirazid and nitazoxanide extracted from myrrh, and other innovative interference directed at the snail, could provide muchneeded alternative chemotherapy. Aggressive monitoring for disease hotspots and control techniques run at animal reservoirs allow early intervention. Simultaneously, increased water and food safety, in conjunction with vaccine production, is crucial for human Fascioliasis prevention strategies. All of this must be followed by vigorous understanding, sensitization, and political support to optimize these measures to be effective.

\section{Conclusion}

Fascioliasis has been recognized as a significant foodborne pathogen with zoonotic and veterinary implications. The advent of drug resistance, the creation of new parasite strains through hybridization and climate change are the major threats that can alter the epidemiology of the disease in the immediate future. To this end, researchers must accelerate their efforts to develop effective vaccinations that provide optimum protection for farm animals and humans while still substantially contributing to the global eradication of the disease by minimizing its occurrence and severity. The governments of tropical and subtropical countries should make deliberate efforts to raise support for research workers.

\section{REFERENCES}

Abbas RZ et al., 2014. Acaricide resistance in cattle ticks and approaches to its management: the state of play. Veterinary Parasitology 203: 6-20.

Ahmad HI et al., 2021. Comparative analysis of the mitochondrial proteins reveals complex structural and functional relationships in Fasciola species. Microbial Pathogenesis 152: 104754.

Ahmed E et al., 2007. Prevalence of Fasciola spp infections of sheep in the Middle awash River Basin, Ethiopia. Southeast Asian Journal of Tropical
Medicine and Public Health 38: 51.

Anderson $\mathrm{N}$ et al., 1999. The sensitivity and specificity of two methods for detecting Fasciola infections in cattle. Veterinary Parasitology 83: 15-24.

Bekele $\mathrm{M}$ et al., 2010. Bovine Fasciolosis. Ethiopian Journal of Applied Science and Technology 1: 39-47.

Bhutto B et al., 2012. Prevalence of Fascioliasis in buffaloes under different agro-climatic areas of Sindh Province of Pakistan. International Journal of Agriculture and Biology 14: 2.

Chemale $\mathrm{G}$ et al., 2010. Comparative proteomic analysis of triclabendazole response in the liver fluke Fasciola hepatica. Journal of Proteome Research 9: 4940-4951.

Chhabra M and Singla L, 2009. Foodborne parasitic zoonoses in India: Review of recent reports of human infections. Journal Veterinary Parasitology 23: 103-110.

Coles $G$ et al., 1986. Anthelmintics for small ruminants. Veterinary Clinics of North America: Food Animal Practice 2: 411-421.

Corvo I et al., 2013. Dissecting the active site of the collagenolytic cathepsin L3 protease of the invasive stage of Fasciola hepatica. PLoS Neglected Tropical Diseases 7: e2269.

Das $M$ et al., 2018. Gastrointestinal parasitic infections in cattle and swamp buffalo of Guwahati, Assam, India. Indian Journal of Animal Research 52: 1732-1738.

Dawes B et al., 1964. Fascioliasis: the invasive stages of Fasciola hepatica in mammalian hosts. Advances in Parasitology 2: 97-168.

Dorny $\mathrm{P}$ et al., 2011. Infections with gastrointestinal nematodes, Fasciola and Paramphistomum in cattle in Cambodia and their association with morbidity parameters. Veterinary Parasitology 175: 293-299.

Dowd AJ et al., 1994. Purification and characterization of a second cathepsin $\mathrm{L}$ proteinase secreted by the parasitic trematode Fasciola hepatica. European Journal of Biochemistry 223, 91-98.

Graczyk TK et al., 2007. Human waterborne trematode and protozoan infections. Advances in Parasitology 64: 111-16o.

$\mathrm{Gu}$ W et al., 2012. Clinical diagnosis and treatment in an outbreak of Fasciola gigantica infection in Yunnan Province. Chinese Journal of Parasitology and Parasitic Diseases 30: 455-459.

Hammami $\mathrm{H}$ et al., 2007. Epidemiological studies on Fasciola hepatica in Gafsa Oases (south west of Tunisia). Parasite 14: 261-264.

Hugh-Jones ME et al., 1995. Zoonoses. Wiley Online Library.

Hurtrez-Boussès S et al., 2001. Dynamics of host-parasite interactions: the example of population biology of the liver fluke (Fasciola hepatica). Microbes and Infection 3: 841-849.

Islam $M$ et al., 2015. Prevalence of fascioliasis in slaughtered goat in Bengal meat abattoir house and its economic impact on business. Journal of Chemical, Biological and Physical Sciences 5: 2684.

Jones KE et al., 2008. Global trends in emerging infectious diseases. Nature 451: 990-993. 
Kadir M et al., 2012. Prevalence of helminthes, pneumonia and hepatitis in Kirkuk slaughter house, Kirkuk, Iraq. Iraqi Journal of Veterinary Sciences, 26(Supplement III): 83-88.

Karim MR et al., 2015. Epidemiological study of bovine fasciolosis: prevalence and risk factor assessment at Shahjadpur Upazila of Bangladesh. Immunology and Infectious Diseases 3: 25-29.

Kelly J et al., 1977. The effect of route of administration on the anthelmintic efficacy of benzimidazole anthelmintics in sheep infected with strains of Haemonchus contortus and Trichostrongylus colubriformis resistant or susceptible to thiabendazole. Research in Veterinary Science 22: 161168.

Kelly $\mathrm{R}$ et al., 2019. Assessing the performance of a Fasciola gigantica serum antibody ELISA to estimate prevalence in cattle in Cameroon. BMC Veterinary Research 15: 1-11.

Khalil-ur-Rehman KJ et al., 2009. Passive surveillance of gastrointestinal parasites in buffaloes of Mandi Bahauddin and Gujrat districts of Punjab. The Journal of Animal and Plant Sciences 19: 17-19.

Khan $\mathrm{K}$ et al., 2018. Impact of agricultural credit on livestock income: A case study of district Lasbela, Balochistan. Sarhad Journal of Agriculture 34: 246250.

Khan $\mathrm{N}$ et al., 2020. Analysis of poverty of different countries of the world. Available at SSRN 3701329.

LaPook JD et al., 20oo. Sheep, watercress and the internet. The Lancet 356: 218.

Le TH et al., 2008. Human fascioliasis and the presence of hybrid/introgressed forms of Fasciola hepatica and Fasciola gigantica in Vietnam. International Journal for Parasitology 38: 725-730.

Lotfy WM et al., 2008. Evolutionary origins, diversification, and biogeography of liver flukes (Digenea, Fasciolidae). The American Journal of Tropical Medicine and Hygiene 79: 248-255.

Maqbool A et al., 2002. Epidemiology of fasciolosis in buffaloes under different managemental conditions. Veterinarski Arhiv 72: 221-228.

Mas-Coma S et al., 2005. Epidemiology of fascioliasis in human endemic areas. Journal of Helminthology 79: 207-216.

Mas-Coma S et al., 2009. Fasciola, lymnaeids and human fascioliasis, with a global overview on disease transmission, epidemiology, evolutionary genetics, molecular epidemiology and control. Advances in Parasitology 69: 41-146.

Hugh-Jones ME et al., 1995. Zoonoses: recognition, control, and prevention. Wiley-Blackwell, pages 384 . DNAL SF740.H84

Mehmood K et al., 2017. A review on epidemiology, global prevalence and economical losses of fasciolosis in ruminants. Microbial Pathogenesis 109: 253-262.

Moje $\mathrm{N}$ et al., 2015. Cross-sectional study on bovine fasciolosis: prevalence, coprological, abattoir survey and financial loss due to liver condemnation at Areka Municipal Abattoir, Southern Ethiopia. Southern
Ethiopian Journal of Veterinary Medicine and Animal Health 7: 33-38.

Mooney L et al., 2009. The comparative efficacy of four anthelmintics against a natural acquired Fasciola hepatica infection in hill sheep flock in the west of Ireland. Veterinary Parasitology 164: 201-205.

Mucheka VT et al., 2015. DNA sequence analyses reveal co-occurrence of novel haplotypes of Fasciola gigantica with $F$. hepatica in South Africa and Zimbabwe. Veterinary Parasitology 214: 144-151.

Mulcahy $G$ et al., 20o1. Cathepsin L proteinases as vaccines against infection with Fasciola hepatica (liver fluke) in ruminants. Research in Veterinary Science 70: 83-86.

Nguyen TG et al., 2009. Genotypic characterization and species identification of Fasciola spp. with implications regarding the isolates infecting goats in Vietnam. Experimental Parasitology 123: 354-361.

Nyindo $\mathrm{M}$ et al., 2015. Fascioliasis: an ongoing zoonotic trematode infection. BioMed Research International, vol. 2015, Article ID 786195, 8 pages, 2015. https://doi.org/10.1155/2015/786195

Opio LG et al., 2021. Prevalence of Fascioliasis and associated economic losses in cattle slaughtered at Lira Municipality Abattoir in Northern Uganda. Animals, 11, 681.

Phiri A et al., 2005. Prevalence of fasciolosis in Zambian cattle observed at selected abattoirs with emphasis on age, sex and origin. Journal of Veterinary Medicine, Series B 52: 414-416.

Qureshi AW et al., 2005. Epidemiology of human fasciolosis in rural areas of Lahore, Pakistan. Punjab University Journal of Zoology 20: 159-168.

Rapsch $C$ et al., 2006. Estimating the true prevalence of Fasciola hepatica in cattle slaughtered in Switzerland in the absence of an absolute diagnostic test. International Journal for Parasitology 36: 1153-1158.

Saleem M et al., 2018. Impact of institutional credit on agriculture production in Pakistan. International Journal of Business Marketing and Management 3: 01-30.

Sanad MM et al., 2005. Fascioliasis among local and imported sheep in Saudi Arabia: parasitological and serological diagnosis. Journal of the Egyptian Society of Parasitology 35: 1121-1134.

Sanchez-Vazquez MJ et al., 2013. Investigating the impact of fasciolosis on cattle carcass performance. Veterinary Parasitology 193: 307-311.

Sargison N et al., 2020. The critical importance of planned small ruminant livestock health and production in addressing global challenges surrounding food production and poverty alleviation. New Zealand Veterinary Journal 68: 136-144.

Schweizer G et al., 2007. Prevalence of Fasciola hepatica in the intermediate host Lymnaea truncatula detected by real time TaqMan PCR in populations from 70 Swiss farms with cattle husbandry. Veterinary Parasitology 150: 164-169.

Tavil B et al., 2014. Severe iron deficiency anemia and marked eosinophilia in adolescent girls with the 
diagnosis of human fascioliasis. The Turkish Journal of Pediatrics 56: 307-309.

Valero MA et al., 2009. Fluke egg characteristics for the diagnosis of human and animal fascioliasis by Fasciola hepatica and F. gigantica. Acta Tropica 111: 150-159.

Vázquez AA et al., 2018. Lymnaeid snails hosts of Fasciola hepatica and Fasciola gigantica (Trematoda: Digenea): a worldwide review. CAB Reviews 13: 1-15.

Walker S et al., 2008. The distribution of Fasciola hepatica and Fasciola gigantica within southern
Tanzania-constraints associated with the intermediate host. Parasitology 135: 495.

Wang C et al., 2006. Survey of helminths in adult sheep in Heilongjiang Province, People's Republic of China. Veterinary Parasitology 140: 378-382.

Youssef A et al., 2014. Review of parasitic zoonoses in Egypt. Tropical Medicine and Health 42: 3-14.

Zafar A et al., 2019. Seroprevalence of Fasciola hepatica in small ruminants of District Chakwal, Punjab, Pakistan. Pakistan Veterinary Journal 39(1). 96-100. 